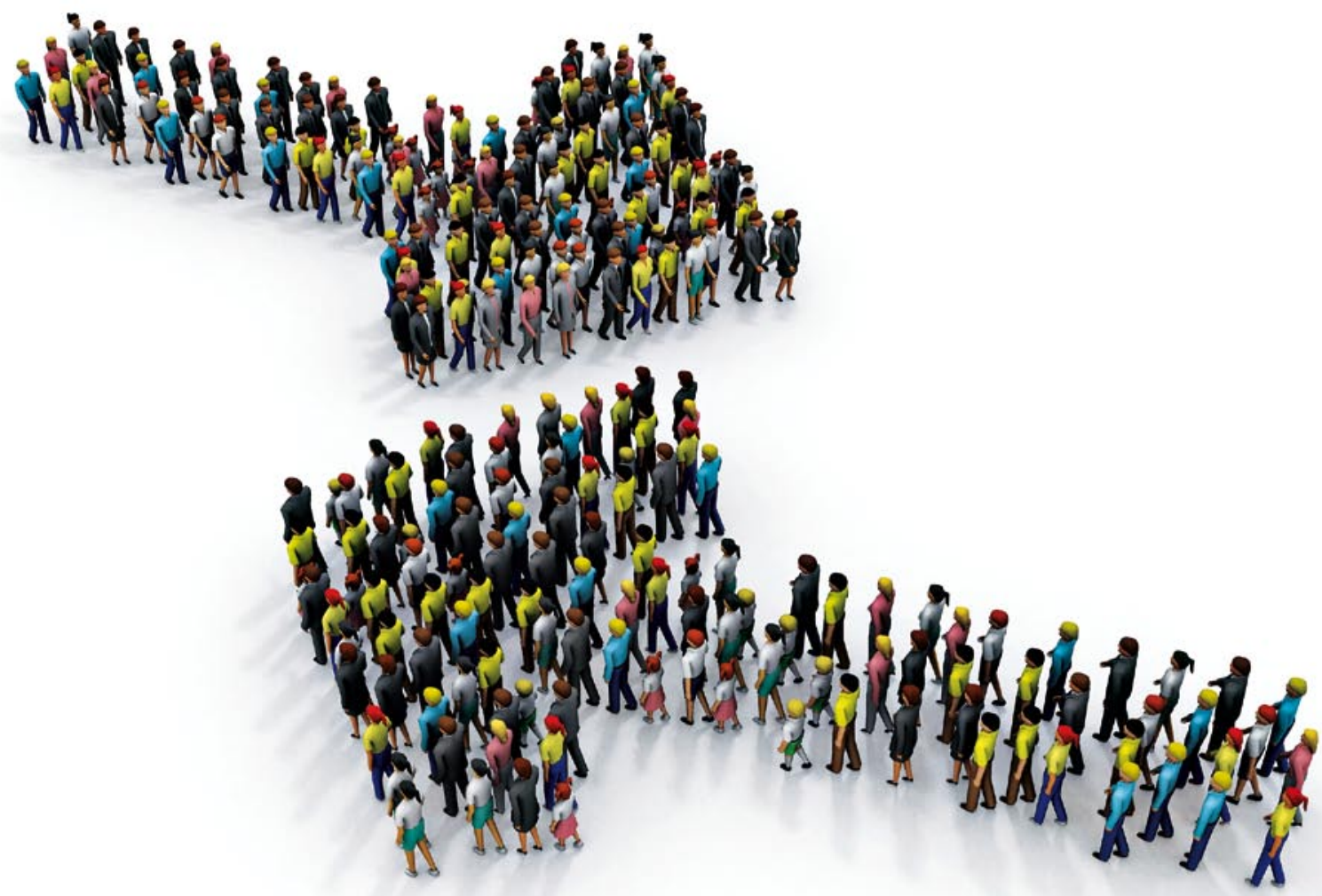

\title{
Kundensegmentierung im Handel - Kaufmotive erkennen und nutzen
}

Konsumenten verhalten sich heute multioptional. Gelingt es Händlern, Einblicke in Kaufmotive ihrer Kunden zu gewinnen, können sie in ihren Sortimenten und Filialen ein klares und unverwechselbares Profil aufbauen und Marketingressourcen gezielt einsetzen. Dieser Beitrag stellt eine Anleitung zur Segmentierung nach Kaufmotiven vor und beschreibt am Beispiel des Textil-, Möbelfach- und Lebensmitteldetailhandels sechs typische Kundensegmente.

Thomas Rudolph | Tillmann Wagner | Timo Sohl

$\mathrm{D}$ Konsumentenverhalten ist durch eine steigende Fragmentierung gekennzeichnet. Eine Vielzahl an Lebens- und Konsumstilen sind Ausprägungen dieser Entwicklung. So lassen sich Konsumenten beispielsweise vor der Kaufentscheidung für einen Fernseher zeitintensiv im Fachhandel beraten und nehmen anschließend kurzerhand noch den Laptop aus der Wochenaktion beim Discounter mit. Kunden verhalten sich bei ihren Kaufentscheidungen selten ausschließlich preis-, produkt- oder serviceorientiert. Denn Bedürfnisse von Konsumenten entstehen häufig situationsspezifisch und sind daher immer weniger mit Durchschnittsbetrachtungen zu erfassen. Vor diesem Hinter- grund stoßen traditionelle Methoden der Kundensegmentierung zunehmend an ihre Grenzen. So spiegeln demografische, typografische und bedürfnisbezogene Eigenschaften der Konsumenten das moderne Kaufverhalten nur unzureichend wider. Vielmehr verschwimmen die Grenzen innerhalb dieser Zielgruppentypologien häufig.

Multioptionale Kunden fordern ein radikales Umdenken. Es müssen Segmentierungskriterien gefunden werden, die das situationsspezifische Kaufverhalten berücksichtigen und bei Konsumentengruppen zentral sind. Psychografische Eigenschaften, wie zum Beispiel Motive oder Einstellungen, scheinen den vielfältigen Verhaltensmustern zugrunde zu liegen (König 2001, S. 21). Neuere Forschungsergebnisse zeigen, dass gerade situativ entstehende Kaufmotive die Kundenwahrnehmung der Warenpräsentation und Einkaufsumgebung maßgeblich beeinflussen (Kaltcheva/Weitz 2006).

Die folgenden Szenarien sollen beispielhaft zwei grundsätzliche Kaufmotive illustrieren. So handelt eine Geschäftsfrau nach einem aufgabenorientierten Kaufmotiv, wenn sie während einer Geschäftsreise schnell ein Geschenk für die Nachbarin kaufen möchte, die ihr während ihrer Abwesenheit die Blumen gießt. Der Produktkauf soll zielgerichtet und ohne großen Aufwand durchzuführen sein. Viele Entscheidungsmöglichkeiten und eine 
komplexe Einkaufsumgebung werden als störend wahrgenommen. Anders stellt sich die Situation dar, wenn die Geschäftsfrau am Samstag vor der Heimreise Zeit für einen Stadtbummel hat und mit dem Gedanken spielt, für sich ein neues Outfit zu kaufen. In diesem Fall genießt sie die Einkaufsaktivität an sich und nimmt Reize in der Einkaufsumgebung positiv wahr. Dem Kaufverhalten der Geschäftsfrau liegt ein erlebnisorientiertes Kaufmotiv zugrunde.

\section{„Bedürfnisse von Konsumenten entstehen häufig situationsspezifisch und sind daher immer weniger mit Durchschnitts- betrachtungen zu erfassen."}

orts mit einer Vielfalt an Angeboten und verstärken somit das Entscheidungsdilemma der Kunden.

Für Marketingmanager drängt sich die Frage auf, wie multioptionale Kunden segmentiert werden sollen und wie ihnen eine optimale Orientierung geboten werden kann. Ziel des vorliegenden Beitrages ist es, zunächst eine Anleitung zur Segmentierung nach Kaufmotiven vorzustellen. Darauf aufbauend können Händler Leistungsangebote ableiten, die ihnen Profil geben und somit ihren Kunden den Weg zur optimalen Produktwahl erleichtern. Der zweite Teil zeigt für die Branchen Textil-,

Je nach Situation verfolgen Konsumenten demnach unterschiedliche Kaufmotive. Das erste Szenario beschreibt mit dem aufgabenorientierten Kaufmotiv einen Zielkauf. Dagegen treffen Konsumenten beim Erlebniskauf häufig ungeplante Kaufentscheidungen, auf die der Händler mit seinem Leistungsangebot Einfluss nehmen kann. Gelingt es Händlern folglich, Einblicke in Kaufmotive ihrer Kunden zu gewinnen, können sie ihre Sortimente und Filialen segmentspezifisch gestalten und Marketingressourcen gezielt einsetzen.

Dass eine Zielgruppenfokussierung heute oft nicht gelingt, zeigt sich auch in dem schwer überschaubaren Waren- und Informationsangebot, das Kunden eine optimale Produktwahl erschwert (Schweizer/Rudolph 2004). Händler reagieren auf die unterschiedlichen Konsumbedürfnisse vieler-
Möbelfach- und Lebensmitteldetailhandel, wie die Segmentierung nach Kaufmotiven eingesetzt werden kann.

\section{Sechs Schritte zur Einkaufsmotiv- Segmentierung}

Kaufmotive stellen die Antriebskräfte der Konsumenten für einen Kauf dar. Sie beeinflussen den Besuch einer bestimmten Verkaufsstelle und das situationsspezifische Kaufverhalten maßgeblich. Zwar sind Kaufmotive in den letzten Jahren stärker in den Mittelpunkt der Segmentierungsliteratur gerückt (Reynolds/Beatty 1999; Ruiz et al. 2004). Es fehlen aber bislang spezifische Handlungsanleitungen, wie die Methodik in der Handelspraxis zu implementieren ist.

Abb. 1 Vorgehen bei der Segmentierung nach Kaufmotiven

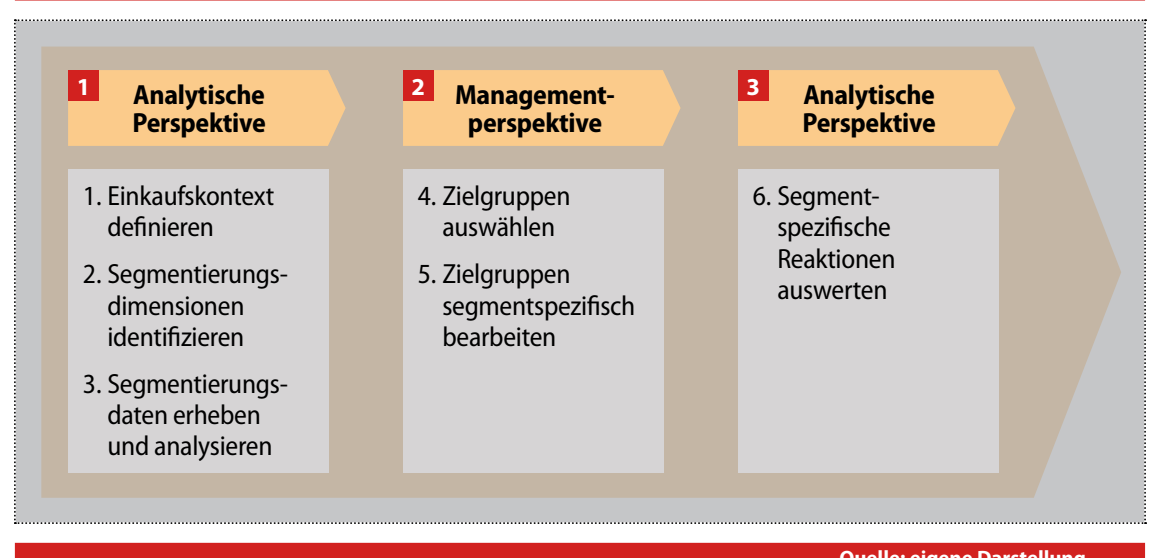

Abbildung 1 zeigt einen dreistufigen Segmentierungsprozess auf, der das Segmentierungsvorgehen in sechs Schritte unterteilt. Dabei wird in Anlehnung an Freter/Obermeier (2002, S. 743) die analytische Perspektive und die Managementperspektive unterschieden. Die analytische Ebene der Kundensegmentierung soll das Konsumentenverhalten durch die Erhebung und Analyse empirischer Daten erklären. Im Zentrum der Managementperspektive stehen dagegen die Zielgruppenauswahl sowie die Formulierung und Implementierung maßgeschneiderter Marketingstrategien.

Der in Abbildung 1 dargestellte Prozess zur Segmentierung nach Kaufmotiven beginnt mit der analytischen Perspektive, die aus den Schritten (1) Einkaufskontext definieren, (2) Segmentierungsvariablen identifizieren und (3) Segmentierungsdaten erheben und analysieren besteht. Darauf folgt in der zweiten Prozessstufe die Managementperspektive mit den Schritten (4) Zielgruppen auswählen und (5) segmentspezifisch bearbeiten. Der Prozess endet mit der analytischen Ebene und dem Schritt (6) segmentspezifische Reaktionen auswerten.

\section{Schritt 1:}

\section{Einkaufskontext definieren}

Die Definition des Einkaufskontextes stellt die Grundlage zur Kaufmotiv-Segmentierung dar und beinhaltet Angebots- und Kundeneigenschaften. Den relevanten Einkaufskontext können Handelsunternehmen mit den fünf Dimensionen Einkaufsaufgabe, Produktkategorie, Handelsformat, Standort und spezifische Situation präzisieren. Eine Einkaufsaufgabe bezeichnet die generelle Einkaufsmission von Konsumenten. Beispiele sind der Kleidungs-, Möbel- oder Lebensmittelkauf. Dagegen repräsentiert eine Produktkategorie einen spezifischeren Teil des Sortimentes, wie zum Beispiel das Jeansangebot. Der relevante Segmentierungskontext kann auch mit Handelsformaten wie ConvenienceLaden oder Online-Händler und dem geografischen Standort präzisiert werden. Dabei grenzt der Standort das Einzugsgebiet eines stationären Händlers ein und enthält somit alle Konsumenten, die in einer bestimmten Gegend wohnen, arbeiten oder diese besuchen. Ein Einkaufskontext kann auch auf eine spezifische Situation, wie beispielsweise das Warten am Flughafen, bezogen sein. 


\section{Schritt 2:}

Segmentierungsdimensionen identifizieren Um die Konsumentenbefragung im nächsten Schritt zielgerichtet durchzuführen, sollten zunächst relevante Segmentierungsdimensionen bestimmt werden. Dabei stellen Dimensionen von Kaufmotiven die Grundlage für die Datenerhebung dar. Zusätzlich können soziodemografische, situationsbezogene und verhaltensbezogene Variablen erfasst werden, um ein besseres Verständnis über die personen- und situationsbezogenen Faktoren innerhalb der Kaufmotivsegmente zu erlangen. Tabelle 1 zeigt häufig verwendete Dimensionen von Kaufmotiven auf und beschreibt das Konsumentenverhalten zu jeder Dimension.

\section{Schritt 3:}

Segmentierungsdaten erheben und analysieren

Kundenbefragungen und -beobachtungen sind in diesem Schritt die wichtigsten Aktivitäten. Mit einem standardisierten Fragebogen, der auf den Segmentierungsdimensionen aufbaut, können Kunden direkt im Laden sowie aufgrund der Kundenkartendaten via Brief und E-Mail kontaktiert werden. Um die Segmentierungsdaten zu analysieren, sollten eine Cluster- und Diskriminanzanalyse durchgeführt werden. Ziel der Clusteranalyse ist die Bündelung aller relevanter Segmentierungsvariablen zu Gruppen. Dabei sollen die Variablen in einer Gruppe möglichst ähnlich und die Gruppen untereinander möglichst verschieden sein. Zur Überprüfung der Ergebnisse aus der Clusteranalyse wird die Diskriminanzanalyse angewendet. Sie untersucht, ob und wie unterschiedliche Variablen zur Unterscheidung zwischen den Gruppen beitragen (zur Durchführung einer Clusterund Diskriminanzanalyse siehe Backhaus et al. 2008). Ziel dieses Schrittes ist es, die Kunden anhand der Kaufmotiv-Dimensionen zu homogenen Gruppen zusammenzufassen.

\section{Schritt 4:}

\section{Zielgruppen auswählen}

Basierend auf den vorangegangenen Schritten zeigen wir ein Tool, das Marketingmanagern bei der Auswahl strategischer Zielgruppen helfen soll. Zur Evaluation der Attraktivität der identifizierten Kundensegmente stellt Abbildung 2 eine SegmentpotenzialMatrix vor. Zunächst werden die einzelnen
Kundensegmente anhand der Dimensionen Segmentwachstum, Segmenterreichbarkeit und Wettbewerbsintensität (Spalte 1) und der daraus abgeleiteten Kriterien (Spalte 2) bewertet. Zur Bewertung der Attraktivität der einzelnen Kaufmotiv-Segmente sind die Werte auf einer Skala (z. B. von 1 = sehr gering bis $5=$ sehr hoch) festzulegen. Zusätzlich können die Werte aufgrund der Relevanz der Kriterien für das Unternehmen gewichtet werden. Die letzten beiden Zeilen der Matrix dienen zur abschließenden Segmentbewertung und Zielgruppenauswahl. Die Segmentattraktivität ergibt sich für jedes Kundensegment aus der Summe der (gewichteten) Punktwerte. Die bewerteten Kundensegmente sollten abschließend noch auf Relevanz für den Zielmarkt des Unternehmens geprüft werden.
Schritt 5:

Zielgruppen segmentspezifisch bearbeiten Die Kenntnis von Kaufmotiven der Zielkunden bildet die Voraussetzung für die strategische Marktbearbeitung. Geschäftsmodelle im Handel sollten daher auf Leistungsversprechen basieren, die auf ein Kaufmotiv oder ein Bündel verwandter Kaufmotive abzielen. So entwickeln einige Händler häufig neue Produkte und präsentieren ihre Sortimente in ansprechenden Läden, um das Kaufverhalten ihrer Kunden anzuregen (z. B. Apple und Zara). Andere sind dagegen mit minimalistisch ausgestatteten Filialen und meist gleichbleibendem Warenangebot erfolgreich (z. B. Aldi und Lidl).

Entsprechend können zur Zielgruppenbearbeitung drei Erfolg versprechende Geschäftsmodelle im Handel - Content Retailer,

\section{Tab. 1 Dimensionen von Kaufmotiven}

\begin{tabular}{|l|l|}
\hline Dimension von Kaufmotiven & Beschreibung des Konsumentenverhaltens \\
\hline Motivation & $\begin{array}{l}\text { Konsumenten verfolgen prinzipiell aufgaben- oder erlebnisorientierte } \\
\text { Kaufmotive }\end{array}$ \\
\hline Rollenerfüllung & $\begin{array}{l}\text { Beschreibt Konsumenten, die ihre Aufmerksamkeit auf den reinen } \\
\text { Produktkauf richten }\end{array}$ \\
\hline Angebotssuche & $\begin{array}{l}\text { Beschreibt Konsumenten, die beim Einkauf eine Rolle erfüllen } \\
\text { (z. B. Mutter, Hausfrau etc.) }\end{array}$ \\
\hline Sozialisierung & $\begin{array}{l}\text { Beschreibt Konsumenten, die auf der Suche nach Angeboten und } \\
\text { Möglichkeiten zur Preisverhandlung sind }\end{array}$ \\
\hline Inspiration & $\begin{array}{l}\text { Beschreibt Konsumenten, die sich beim Einkauf mit Freunden und } \\
\text { Familie treffen oder gerne vom Verkaufspersonal bedient werden }\end{array}$ \\
\hline Stimulation & $\begin{array}{l}\text { Beschreibt Konsumenten, die nach neuen Ideen und Trends } \\
\text { suchen }\end{array}$ \\
\hline $\begin{array}{l}\text { Beschreibt Konsumenten, die sich aufregende Einkaufsumgebungen } \\
\text { wünschen }\end{array}$
\end{tabular}

In Anlehnung an: Westbrook/Black 1985; Kaltcheva/Weitz 2006

Abb. 2 Segmentpotenzial-Matrix

\begin{tabular}{|c|c|c|c|c|}
\hline & Kriterien & $\begin{array}{l}\text { Kunden- } \\
\text { segment } 1\end{array}$ & $\ldots$ & $\begin{array}{l}\text { Kunden- } \\
\text { segment } n\end{array}$ \\
\hline \multirow{3}{*}{$\begin{array}{l}\text { Segmentwachstum } \\
\text { Was ist das Umsatz- } \\
\text { potenzial der Segmente? }\end{array}$} & Heutiger Umsatzanteil & & & \\
\hline & Wachstumsrate & & & \\
\hline & Segmentpotenzial & & & \\
\hline \multirow{3}{*}{$\begin{array}{l}\text { Segmenterreichbarkeit } \\
\text { Verfügt das Unterneh- } \\
\text { men über die notwen- } \\
\text { digen Ressourcen? }\end{array}$} & Kompetenzfit & & & \\
\hline & Finanzielle Ressourcen & & & \\
\hline & $\begin{array}{l}\text { Fit mit Unternehmens- } \\
\text { kultur }\end{array}$ & & & \\
\hline \multirow{3}{*}{$\begin{array}{l}\text { Wettbewerbsintensität } \\
\text { Wie stark ist die Wett- } \\
\text { bewerbsintensität? }\end{array}$} & Anzahl Wettbewerber & & & \\
\hline & Markteintrittsbarrieren & & & \\
\hline & Konkurrenzvorsprung & & & \\
\hline \multicolumn{5}{|l|}{ 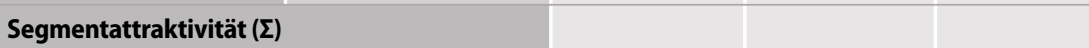 } \\
\hline Zielmarktrelevanz & & & & \\
\hline
\end{tabular}


Channel Retailer und Global Discounter unterschieden werden (Rudolph 2000). So bieten Unternehmen wie Apple und Zara einzigartige Eigenmarkenprodukte in einer ansprechenden Einkaufsumgebung an. Zum InStore-Erlebnis trägt auch die entsprechende Wahl von Musik, Farben und Beleuchtung bei. Handelsunternehmen dieser Kategorie zählen zu Content Retailern. Dagegen zielt das Leistungsversprechen der Channel Retailer auf ein inspirierendes und zugleich bequemes Einkaufen ab. Die Reizauslösung in den Filialen fällt daher deutlich geringer aus. Beispiele für Channel Retailer sind die Handelsunternehmen Metro C\&C und Wal-Mart. Global Discounter wie Aldi und Lidl stehen für dauerhaft tiefe Preise und schnelle Einkaufsmöglichkeiten. Global Discounter vermeiden folglich jegliche Reize und Komplexität in der Einkaufsumgebung.

\section{Schritt 6:}

Segmentspezifische Reaktionen auswerten Der Segmentierungsprozess hört nicht mit der Zielgruppenbearbeitung auf. Im letzten Schritt sollten die Kundenreaktionen auf die segmentspezifische Marktbearbeitung analy- siert werden. Dazu dienen einerseits leicht auswertbare Daten wie Umsatzentwicklungen und Wiederkaufsraten. Zusätzlich helfen Kundenzufriedenheitsmessungen, bestehende Kaufbarrieren der Zielgruppen zu identifizieren und weitere Maßnahmen zur Optimierung des Einkaufsprozesses abzuleiten.

\section{Empirische Untersuchung}

Die vorangegangene Prozessbeschreibung stellte die Segmentierung nach Kaufmotiven vor. Dieser Abschnitt zeigt die Anwendung der Methodik mit den Ergebnissen einer branchenübergreifenden empirischen Untersuchung auf. Die Ergebnisse basieren auf einer standardisierten schriftlichen Kundenbefragung, die in den Filialen eines Textil-, Möbelfach- und Lebensmitteldetailhändlers wochentags und an Samstagen durchgeführt wurde. Insgesamt haben 707 zufällig ausgewählte Konsumenten den Fragebogen vollständig ausgefüllt. Davon sind 335 Fragebögen beim Lebensmitteleinkauf und 372 beim Non-Food-Einkauf ( $\mathrm{N}=210$ Textil und N=162 Möbel) ausgefüllt worden. Zur Seg-

\section{Abb. 3 Segmentierungs-Matrix Textilhandel}

\begin{tabular}{|c|c|c|c|c|c|}
\hline & & $\begin{array}{l}\text { Freizeit- } \\
\text { käufer } \\
34 \%(\mathrm{~N}=67)\end{array}$ & $\begin{array}{l}\text { Pflicht- } \\
\text { käufer } \\
23 \%(\mathrm{~N}=46)\end{array}$ & $\begin{array}{l}\text { Einkaufs- } \\
\text { enthusiasten } \\
22 \%(N=43)\end{array}$ & $\begin{array}{l}\text { Haushalts- } \\
\text { käufer } \\
21 \%(\mathrm{~N}=41)\end{array}$ \\
\hline \multirow{7}{*}{ 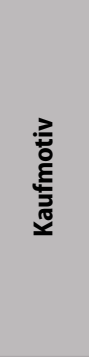 } & $\begin{array}{l}\text { Motive }{ }^{1} \text { (aufgaben- } \\
\text { vs. erlebnisorientiert) }\end{array}$ & + & - & ++ & 0 \\
\hline & Produkterwerb ${ }^{1}$ & 0 & + & + & ++ \\
\hline & Rollenerfüllung ${ }^{1}$ & - & -- & ++ & ++ \\
\hline & Angebotssuche $^{1}$ & + & 0 & ++ & + \\
\hline & Sozialisierung ${ }^{1}$ & - & -- & + & -- \\
\hline & Inspiration ${ }^{1}$ & + & - & ++ & 0 \\
\hline & Stimulation ${ }^{1}$ & - & -- & + & -- \\
\hline \multirow{6}{*}{ 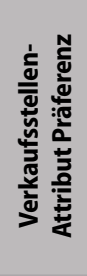 } & Verkaufsstelle ${ }^{1}$ & ++ & + & +++ & ++ \\
\hline & Preis ${ }^{1}$ & ++ & + & ++ & ++ \\
\hline & Produkt $^{1}$ & + & 0 & ++ & + \\
\hline & Personal $^{3}$ & +++ & +++ & +++ & +++ \\
\hline & Service $^{1}$ & ++ & + & +++ & ++ \\
\hline & Erreichbarkeit ${ }^{1}$ & ++ & + & ++ & +++ \\
\hline \multicolumn{6}{|c|}{ Legende: --- sehr niedrig; -- niedrig; - eher niedrig; 0 neutral; + eher hoch; ++ hoch; +++ sehr hock } \\
\hline \multicolumn{6}{|c|}{$\begin{array}{l}{ }^{1} p<.05 \\
{ }^{2} \text { hoch entspricht erlebnisorientiert, niedrig entspricht aufgabenorientiert } \\
{ }^{3} \text { Die Verkaufsstellen-Attribut Präferenz zeigt für den Faktor Personal keine signifikanten Unterschiede zwischen den Clustern } \\
\text { auf. Beim Textilkauf haben Konsumenten generell ein hohes Verlangen nach freundlichem und kompetentem Verkaufs- } \\
\text { personal. Folglich ist die Variable Personal für jedes der vier Kundensegmente von hoher Bedeutung und kann deshalb } \\
\text { nicht zur Erklärung von Gruppenunterschieden herangezogen werden. }\end{array}$} \\
\hline
\end{tabular}

mentbildung wurde der dreistufige Segmentierungsprozess aus Abbildung 1 angewendet. Zusätzlich zu den Kaufmotiven und Verkaufsstellenpräferenzen wurden passive Variablen wie Einkommen, Geschlecht und Alter erhoben. Die Abbildungen 3, 4 und 5 zeigen die Ergebnisse der Untersuchung auf.

Nach Abbildung 3 können im Textilhandel die Kaufmotive von vier Kundensegmenten, Freizeitkäufer, Pflichtkäufer, EinkaufsEnthusiasten und Haushaltskäufer, unterschieden werden.

Freizeitkäufer repräsentieren mit $34 \%$ das größte Kundensegment im Textilhandel. Konsumenten dieses Segments genießen die Einkaufsaktivität an sich (erlebnisorientierte Motivation). Sie suchen nach Inspiration und legen großen Wert darauf, sich bei der Shoppingaktivität wohl zu fühlen. Freizeitkäufer besuchen die Filiale eines Händlers nicht für den gezielten Produkterwerb, sondern treffen ihre Kaufentscheidungen meist spontan. Neben dem Personal, das für alle Segmente im Textilhandel wichtig ist, kommt den Faktoren Verkaufsstelle, Preis, Service und Erreichbarkeit für Freizeitkäufer eine große Bedeutung zu. Das Kundensegment setzt sich überwiegend aus jüngeren (30 Jahre und jünger) sowie älteren (61 Jahre und älter) Kunden zusammen.

Pflichtkäufer machen fast ein Viertel der befragten Textilkunden aus. Sie empfinden den Einkauf als eine Zwangsaufgabe, die sie nach Möglichkeit zu vermeiden versuchen. Ihrem Einkauf liegt ein aufgabenorientiertes Kaufmotiv zugrunde. Entsprechend stellt bei diesem Kundensegment der Produkterwerb das alleinige Motiv für den Einkaufsgang dar. Pflichtkäufer bevorzugen folglich Verkaufsstellen von Händlern, in denen die Produktakquisition mit einem minimalen kognitiven und zeitlichen Aufwand durchzuführen ist. Das Kundensegment besteht überwiegend aus 51- bis 60-Jährigen.

Einkaufsenthusiasten sind mit $22 \%$ beim Textilkauf relativ stark repräsentiert. Wie kein anderes Kundensegment empfinden sie die Einkaufsaktivität an sich als Vergnügen. Bei ihnen dominieren hedonistische und erlebnisorientierte Kaufmotive. Im Gegensatz zu Freizeitkäufern, die ebenso erlebnisorientierte Kaufmotive verfolgen, haben Einkaufsenthusiasten aber ein klares Einkaufsziel vor Augen. Einkaufsenthusiasten sind eine anspruchs- 
volle Klientel. Dies zeigt sich auch in ihrer Präferenzstruktur der Verkaufsstellen-Attribute, von denen alle sechs Faktoren für sie wichtig sind. Folglich ist es für Händler eine Herausforderung, diese Kundengruppe zufriedenzustellen. Das kann sich bei gezielter Kundenbearbeitung aber durchaus als Chance herausstellen, wie einige Händler beweisen.

Haushaltskäufer sind mit $20 \%$ im Textilhandel repräsentiert. Sie akzeptieren die Notwendigkeit des Einkaufs und nehmen diesen weder positiv noch negativ wahr. Eine soziale oder familiäre Rolle zu erfüllen und der Produkterwerb als solcher stellen die zentralen Ziele ihrer Einkaufsaktivitäten dar. Daneben ist die Suche nach Angeboten ein weiteres, wenn auch weniger stark ausgeprägtes Kaufmotiv. Im Hinblick auf die VerkaufsstellenAttribute haben die Faktoren Erreichbarkeit und Personal den stärksten Einfluss auf die Präferenz bei der Händlerwahl. Auch die Faktoren Preis, Filiale und Service sind wichtige Entscheidungskriterien der Haushaltskäufer. Die 41- bis 50-Jährigen sind die dominierende Altersgruppe in diesem Segment und die Haushaltsgröße beträgt überwiegend fünf oder mehr Personen.

Abbildung 4 zeigt die Ergebnisse der Segmentierung nach Kaufmotiven für den Möbelfachhandel auf. Auch hier konnten wie beim Textilhandel die Segmente Haushaltskäufer und Pflichtkäufer identifiziert werden. Eine zusätzliche Kundengruppe ergibt sich aber beim Möbelkauf mit dem Segment der Ideensucher.

Ideensucher repräsentieren mit fast der Hälfte aller Kunden das größte Kaufmotivsegment im Möbelfachhandel. Die Kundengruppe der Ideensucher genießt es, sich in den Handelsfilialen aufzuhalten. Sie kommt in die Filiale, um sich inspirieren zu lassen, und hat nicht notwendigerweise den Erwerb eines bestimmten Produktes geplant. Frauen sind im Segment der Ideensucher weit überdurchschnittlich vertreten.

Haushaltskäufer stellen mit rund einem Drittel die zweitgrößte Kundengruppe nach Kaufmotiven im Möbelfachhandel dar. Im Gegensatz zu ihrer neutralen Motivation (aufgaben- vs. erlebnisorientiert) im Textilhandel verfolgen Haushaltskäufer im Möbelfachhandel tendenziell erlebnisorientierte Motive. Ein weiterer Unterschied zeigt sich darin, dass die Kundengruppe der Haushalts- käufer beim Möbelkauf überwiegend aus Männern besteht.

Pflichtkäufer machen ein Viertel der Möbelfachhandelskunden aus. Auch beim Möbelkauf verfolgen Pflichtkäufer aufgabenorientierte Motive und tendieren dazu, die Einkaufsaktivität an sich als unangenehm zu empfinden. Für Pflichtkäufer stellt der Produkterwerb den einzigen Grund dar, eine Handelsfiliale aufzusuchen.

In Abbildung 5 werden für den Lebensmitteldetailhandel drei Kaufmotiv-Segmente unterschieden. Das Kundensegment der Pflichtkäufer trat schon in den beiden zuvor diskutierten Einkaufskontexten auf. Auch die Kundengruppe der Einkaufsenthusiasten wurde bereits im Kontext des Textilhandels vorgestellt. Neu ist das Segment der indifferenten Käufer.

Indifferente Käufer kommen unter den Kaufmotivsegmenten im Lebensmitteldetailhandel auf einen Anteil von 40 \%. Sie können nach ihrer Einkaufsorientierung (aufgaben- vs. erlebnisorientiert) als neutral charakterisiert werden. Sie stehen auch der Einkaufsaktivität als solcher neutral gegenüber. Der effiziente Erwerb von Produkten des täglichen Bedarfs und die Suche nach Angeboten stellen die zentralen
Motive dar. Konsumenten aus diesem Segment verhalten sich entsprechend preissensibel.

Pflichtkäufer kommen wie das Segment der indifferenten Käufer auf einen Anteil von 40 \%. Pflichtkäufer empfinden die Einkaufsaktivität wie in den bereits beschriebenen Einkaufskontexten als notwendig, aber unangenehm. Die meisten Konsumenten aus der Gruppe der Pflichtkäufer sind 30-jährige oder jüngere Kunden und der empfundene Zeitdruck ist beim Lebensmitteleinkauf vergleichsweise am höchsten.

Einkaufsenthusiasten repräsentieren ein Fünftel der Kunden im Lebensmitteldetailhandel. Zwar verhalten sich Konsumenten beim Lebensmittelkauf überwiegend utilitaristisch. Dennoch verfolgen Konsumenten aus diesem Segment erlebnisorientierte Motive, was vor allem auf das bewusste Ernährungsverhalten dieser Zielgruppe zurückzuführen ist. So geben sich Einkaufsenthusiasten auch beim Lebensmitteleinkauf als eine sehr anspruchsvolle und wählerische Kundschaft.

Die Zielsetzung der Segmentierung nach Kaufmotiven besteht darin, Kunden vor dem Hintergrund des Einkaufskontextes in homogene Gruppen zu überführen. Unter Berücksichtigung der Branchenbesonderheiten des

\section{Abb. 4 Segmentierungs-Matrix Möbelfachhandel}

\begin{tabular}{|c|c|c|c|c|}
\hline & & $\begin{array}{l}\text { Ideensucher } \\
45 \%(\mathrm{~N}=67) \\
\end{array}$ & $\begin{array}{l}\text { Haushaltskäufer } \\
30 \%(\mathrm{~N}=46)\end{array}$ & $\begin{array}{l}\text { Pflichtkäufer } \\
25 \%(\mathrm{~N}=37)\end{array}$ \\
\hline \multirow{7}{*}{ 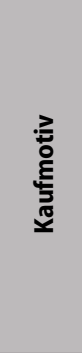 } & $\begin{array}{l}\text { Motive }{ }^{1} \text { (aufgaben- } \\
\text { vs. erlebnisorientiert) }\end{array}$ & ++ & + & - \\
\hline & Produkterwerb ${ }^{1}$ & - & + & +++ \\
\hline & Rollenerfüllung ${ }^{1}$ & - & ++ & -- \\
\hline & Angebotssuche $^{1}$ & - & + & - \\
\hline & Sozialisierung ${ }^{1}$ & -- & -- & --- \\
\hline & Inspiration ${ }^{1}$ & ++ & + & 0 \\
\hline & Stimulation ${ }^{1}$ & + & - & -- \\
\hline \multirow{6}{*}{ 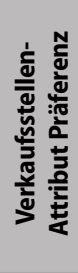 } & Verkaufsstelle ${ }^{1}$ & +++ & ++ & ++ \\
\hline & Preis $^{3}$ & + & + & + \\
\hline & Produkt $^{3}$ & + & + & + \\
\hline & Personal ${ }^{1}$ & +++ & +++ & +++ \\
\hline & Service $^{1}$ & ++ & + & + \\
\hline & Erreichbarkeit ${ }^{1}$ & +++ & +++ & ++ \\
\hline \multicolumn{5}{|c|}{ Legende: --- sehr niedrig; -- niedrig; - eher niedrig; 0 neutral; + eher hoch; ++ hoch; +++ sehr hoch } \\
\hline \multicolumn{5}{|c|}{$\begin{array}{l}{ }^{1} \mathrm{p}<.05 \\
{ }^{2} \text { hoch entspricht erlebnisorientiert, niedrig entspricht aufgabenorientiert } \\
{ }^{3} \text { Die Verkaufsstellen-Attribut Präferenz zeigt für die Faktoren Produkt und Preis keine signifikanten Unterschiede zwischen } \\
\text { den Kundensegmenten auf. Diesen Faktoren kommt daher bei der Erklärung der unterschiedlichen Cluster nur eine be- } \\
\text { grenzte Aussagekraft zu. }\end{array}$} \\
\hline
\end{tabular}


Textil-, Möbelfach- und Lebensmitteldetailhandels konnten so insgesamt sechs Kundensegmente identifiziert werden. Die drei KaufmotivSegmente Pflichtkäufer, Einkaufsenthusiasten und Haushaltskäufer zeigen dabei branchenunabhängig große Ähnlichkeiten auf.

\section{„Händler sollten sich auf ausgewählte Kaufmotive spezialisieren und diese besser als die Konkurrenz bearbeiten.“}

Fazit

Es bleibt festzuhalten, dass Konsumenten beim Produkterwerb je nach Situation unterschiedliche Faktoren wichtig sind. So verfolgen dieselben Konsumenten je nach Situation auch unterschiedliche Kaufmotive. Oft reagieren Händler auf das multioptionale Kundenverhalten mit dem Ziel, alles jederzeit für jeden bieten zu wollen. Durch die gleichzeitige Anspra-

Um erfolgreich zu sein, sollten sich Händler auf ausgewählte Kaufmotive spezialisieren und diese besser als die Konkurrenz bearbeiten. So spricht beispielsweise der Möbelhändler Pfister mit seinen inspirierenden Verkaufsstellen und intensiven Beratungsleistungen das Segment der Ideensucher an. Kunden werden sofort angesprochen und zeitaufwändig beraten, wenn sie eine Filiale betreten. Dagegen konzentriert sich Konkurrent Ikea auf die Segmente der Haushalts- und Pflichtkäufer. Der erste Kontakt mit Ikea-Personal findet daher häufig erst an der Kasse statt. Beide Unternehmen haben durch ihre Fokussierung ihr Profil geschärft und eine eindeutige Wettbewerbsposition eingenommen. che unterschiedlicher Kaufmotive werden aber die jeweiligen Erwartungen von Kundengruppen immer nur teilweise oder gar nicht erfüllt. Das kann die Zufriedenheit der Kunden beim Einkauf erheblich beeinträchtigen.

Vielmehr sollten zur zielgerichteten Marktbearbeitung relevante Cluster von Kaufmotiven erkannt werden. Erfolgreiche Händler haben sich vom Massenmarketing abgewandt und fokussieren ihr Leistungsversprechen auf verwandte Kaufmotive. So bauen sie ein klares und unverwechselbares Profil auf. Bei diesen Händlern verkörpert die konsequente Bearbeitung von ausgewählten Kaufmotiven das Gravitationsfeld für die Kundenbesuche in ihren Verkaufsstellen.

\section{Literatur}

Backhaus, K./Erichson, B./Plinke, W./Weiber, R. (2008): Multivariate Analysemethoden: Eine anwendungsorientierte Einführung, 12., vollständig überarb. Aufl., Berlin.

Freter, H./Obermeier, O. (2002): Marktsegmentierung, in: Hermann, A./Homburg, C. (Hrsg.): Marktforschung: Methoden, Anwendungen, Praxisbeispiele, Wiesbaden, S. 739-763.

Kaltcheva, V. D./Weitz, B. A. (2006): When Should a Retailer Create an Exciting Store Environment?, in: Journal of Marketing, 70, January, S. 107-118.

König, T. (2001): Nutzensegmentierung und alternative Segmentierungsansätze, Wiesbaden.

Reynolds, K. E./Beatty, S. E. (1999): A Relationship Customer Typology, in: Journal of Retailing, 75, 4, S. 509-523.

Rudolph, T. (2000): Erfolgreiche Geschäftsmodelle im europäischen Handel: Ausmaß, Formen und Konsequenzen der Internationalisierung für das Handelsmanagement, in: Thexis 2000/3.

Rudolph, T. (2005): Modernes Handelsmanagement, München.

Ruiz, J. P./Chebat, J. C./Hansen, P. (2004): Another trip to the mall: a segmentation study of consumers based on their activities, in: Journal of Retailing and Consumer Services, 11, S. 333-359.

Schweizer, M./Rudolph, T. (2004): Wenn Käufer streiken: Mit klarem Profil gegen Consumer Confusion und Kaufmüdigkeit, Wiesbaden.

Westbrook, R. A./Black, W. C. (1985): A MotivationBased Shopper Typology, in: Journal of Retailing, 61, 1, S. 78-103.

\section{Abb. 5 Segmentierungs-Matrix Lebensmitteldetailhandel}

\begin{tabular}{|c|c|c|c|c|}
\hline & & $\begin{array}{l}\text { Indifferente Käufer } \\
40 \%(\mathrm{~N}=130)\end{array}$ & $\begin{array}{l}\text { Pflichtkäufer } \\
40 \% \text { ( } N=129)\end{array}$ & $\begin{array}{l}\text { Einkaufsenthusiasten } \\
20 \%(\mathrm{~N}=63)\end{array}$ \\
\hline \multirow{7}{*}{ 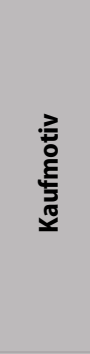 } & $\begin{array}{l}\text { Motive' (aufgaben- } \\
\text { vs. erlebnisorientiert) }{ }^{2}\end{array}$ & 0 & -- & ++ \\
\hline & Produkterwerb ${ }^{1}$ & ++ & +++ & ++ \\
\hline & Rollenerfüllung ${ }^{1}$ & 0 & - & ++ \\
\hline & Angebotssuche ${ }^{1}$ & ++ & 0 & ++ \\
\hline & Sozialisierung ${ }^{1}$ & -- & --- & 0 \\
\hline & Inspiration ${ }^{1}$ & 0 & -- & ++ \\
\hline & Stimulation ${ }^{1}$ & - & --- & + \\
\hline \multirow{6}{*}{ 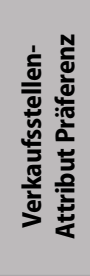 } & Verkaufsstelle ${ }^{1}$ & ++ & + & +++ \\
\hline & Preis ${ }^{1}$ & ++ & + & +++ \\
\hline & Produkt $^{1}$ & + & 0 & ++ \\
\hline & Personal ${ }^{1}$ & ++ & ++ & +++ \\
\hline & Service $^{1}$ & ++ & ++ & ++ \\
\hline & Erreichbarkeit ${ }^{1}$ & + & + & +++ \\
\hline \multicolumn{5}{|c|}{ Legende: --- sehr niedrig; -- niedrig; - eher niedrig; 0 neutral; + eher hoch; ++ hoch; +++ sehr hoch } \\
\hline \multicolumn{5}{|c|}{$\begin{array}{l}1 \mathrm{p}<.05 \\
{ }^{2} \text { hoch entspricht erlebnisorientiert, niedrig entspricht aufgabenorientiert }\end{array}$} \\
\hline
\end{tabular}

\section{Autoren}

\section{Prof. Dr. Thomas Rudolph}

Direktor des Forschungszentrums für Handelsmanagement der Universität St.Gallen, Leiter des Gottlieb Duttweiler Lehrstuhls für Internationales Handelsmanagement und des Retail-Lab.

E-Mail:

thomas.rudolph@unisg.ch

\section{Dr. Tillmann Wagner}

Assistant Professor of Marketing at Rawls College of Business, Texas Tech University. E-Mail:

t.wagner@ttu.edu

\section{Dipl.-Volksw. Timo Sohl}

Wissenschaftlicher Mitarbeiter am Forschungszentrum für Handelsmanagement der Universität St. Gallen.

E-Mail:

timo.sohl@unisg.ch 